\title{
What is the price of life?
}

\author{
Márcio Galindo Kiuchi ${ }^{1,2}$ \\ ${ }^{1}$ Division of Artificial Cardiac Stimulation, Department of Medicine, Hospital e Clínica São Gonçalo, São Gonçalo, RJ, Brazil \\ ${ }^{2}$ Department of Cardiology, Elisabethinen University Teaching Hospital Linz, Linz, Austria
}

Recently, the St. Jude Medical (SJM) notified that its records indicate that patients who have an implantable device, automatic implantable cardioverter defibrillators (ICD's) [1] and implantable resynchronizing defibrillators (CRT-D's) [2] manufactured before May 2015 may be subject to problems. The company claims that the likelihood that this will impact the health of each patient is low since the vast majority of devices do not present premature battery depletion. Currently, out of a total of 398,740 devices sold worldwide that are subject to this release, 841 devices $(0.21 \%)$ have returned to SJM with premature battery wear. Note that, in general, the complaints made to SJM are sometimes underestimated and that this rate may change. The cause of premature battery depletion is attributed to the formation of lithium agglomerates (deposits) inside the battery, causing a dead short. There is a possibility that affected devices may lose battery power within days. So far, there have been two deaths and ten major events (syncope) that may have been associated with premature battery depletion.

From the top of my estate, based on logical reasoning and in my opinion from Professor Doctor and Specialist in Artificial Cardiac Stimulation, SJM is playing with patients' lives. In view of the facts presented in the communique presented by the aforementioned company, I request that the device of each patient be changed in an emergency by a device with the same functions, but from another manufacturer. Besides, who guarantees us that there is only one defective lot? For in the face of an act of such bad faith, all things are possible. I propose THREE hypothetical scenarios:

1. A patient with sinus dysfunction and an implantable cardioverter defibrillator (ICD) presented $10 \%$ atrial pacing $(\mathrm{AP}=10 \%)$ and $<1 \%$ ventricular pacing $(\mathrm{VP}<1 \%)$, with battery longevity of 5 years, without arrhythmic events. And your next review is scheduled for 3 or 4 months from now. If during this interval premature battery drainage occurs and the patient requires the functions of "anti-tachycardia pacing" (ATP) or shock, there will not be enough battery to perform these functions, leading to death;

2. A patient with a total atrioventricular block and an implantable cardioverter-defibrillator (ICD) presented 5\% atrial pacing $(\mathrm{AP}=5 \%)$ and $>99 \%$ ventricular pacing $(\mathrm{VP}>$ $99 \%$ ), with battery longevity of 5.3 years, without arrhythmic events. And your next review is scheduled for 3 or 4 months from now. If during this interval premature battery drainage occurs, the patient may experience syncope and dyspnea, even prolongation of the QT interval, triggering episodes of sustained ventricular tachycardia "Torsades de Pointes," degenerating to ventricular fibrillation and sudden death. If due to the same or another ventricular tachyarrhythmia, there is a need for anti-tachycardia pacing (ATP) or shock, there will not be enough battery to perform these functions, leading to death;

3. A patient with sinus dysfunction and distal block (96 ms HV interval) with implantable cardioverter defibrillator (ICD) presented $55 \%$ atrial pacing $(\mathrm{AP}=55 \%)$ and $68 \%$ pacing Ventricular pacing "(VP $=68 \%)$, with a battery longevity of 4.7 years, without arrhythmic events. And your next review is scheduled for 3 or 4 months from now. If during this interval premature battery drainage occurs, the patient may experience syncope and dyspnea, even prolongation of the QT interval, triggering episodes of sustained ventricular tachycardia "Torsades de Pointes," degenerating to ventricular fibrillation and sudden death. If due to the same or another ventricular tachyarrhythmia, there is a need for anti-tachycardia pacing (ATP) or shock, there will not be enough battery to perform these functions, leading to death. There is also the hypothesis that a short circuit is generated from the system, generating, for example, 83 inappropriate shocks leading to acute battery exhaustion and myocardial stunning, due to the cumulative joules of each shock, leading to death.

In addition to these three hypothetical scenarios, there are many others that lead to death. We could spend a day reporting such. It is worth noting that the vast majority of these patients have a low left ventricular ejection fraction, have ischemic coronary artery disease, and have a myocardial infarction, hypertrophic cardiomyopathy, Brugada syndrome, among other serious conditions. I can state everything I write categorically because I am one of the doctors with the largest number of St. Jude Medical ICD's and CRT-D's implanted in Brazil. However, when the problem appears we should solve it and not "push it down the carpet." Everything is time, time is nothing, nothing is everything, and time is everything. How much does a life cost?

Correspondence to: Márcio Galindo Kiuchi, Rua Cel. Moreira César, 138 Centro, São Gonçalo - Rio de Janeiro - Brazil. ZIP-CODE: 24440-400, Tel: +55 (21) 26047744; E-mail: marciokiuchi@gmail.com

Received: June 19, 2017; Accepted: July 24, 2017; Published: July 27, 2017 


\section{References}

1. Priori SG, Blomström-Lundqvist C, Mazzanti A, Blom N, Borggrefe M, et al. (2015) 2015 ESC Guidelines for the management of patients with ventricular arrhythmias and the prevention of sudden cardiac death: The Task Force for the Management of Patients with Ventricular Arrhythmias and the Prevention of Sudden Cardiac Death of the European Society of Cardiology (ESC). Endorsed by: Association for European Paediatric and Congenital Cardiology (AEPC). Eur Heart J 36: 2793-2867. [Crossref]
2. European Society of Cardiology (ESC), European Heart Rhythm Association (EHRA), Brignole M, Auricchio A, Baron-Esquivias G, et al. (2013) 2013 ESC guidelines on cardiac pacing and cardiac resynchronization therapy: the task force on cardiac pacing and resynchronization therapy of the European Society of Cardiology (ESC) Developed in collaboration with the European Heart Rhythm Association (EHRA) Europace 15: 1070-1118. [Crossref]

Copyright: (C2017 Kiuchi MG. This is an open-access article distributed under the terms of the Creative Commons Attribution License, which permits unrestricted use, distribution, and reproduction in any medium, provided the original author and source are credited. 